\title{
Haptic curvature discrimination at several regions of the hand
}

\author{
SYLVIA C. PONT, ASTRID M. L. KAPPERS, and JAN J. KOENDERINK \\ Helmholtz Instituut, Utrecht, The Netherlands
}

\begin{abstract}
Static haptic discrimination of the curvature of convex, concave, or straight 20 -cm-long strips was investigated for nine placements on the hand. In one condition, the strips were touched with the palmar side of the hand, and in the other condition, with the dorsal side. The influence of the lengths of the strips, and thus of contact lengths, was also investigated. For all placements, discrimination was poorer in the dorsal than in the palmar condition, owing to poorer cutaneous resolution on the dorsal side of the hand (the kinesthetic stimulation was the same in both conditions). Thus cutaneous stimulation is important. In both experiments, performance appeared to depend primarily on contact length. Moreover, the discrimination thresholds for all different placements and contact lengths followed the same trend. We conclude that in these experiments the effective stimulus for the discrimination of curved strips is the total difference of local surface attitude-that is, the slope difference over the far ends of the stimulus.
\end{abstract}

Our research aims to investigate static haptic discrimination of the curvature of surfaces in a quantitative way [the curvature is the reciprocal of the radius of curvature and vice versa, so a curvature of $k=0.8 / \mathrm{m}$ is represented by a circle with a radius of $(1 / 0.8) \mathrm{m}=1.25 \mathrm{~m}]$. We want to gain insight into the various roles played by different parts of the hand. With mathematically well-defined forms consisting of $20-\mathrm{cm}$-long and $2-\mathrm{cm}$-wide strips of constant curvature, we systematically vary the geometrical structure available to the haptic system.

We are interested in finding out whether the structure of the part of the hand with which the stimuli are touched has an influence on the sensitivity for curvature discrimination. Different parts of the hand contain various kinds and numbers of bones and joints. Also, the hand tissue varies largely from place to place. This heterogeneity seems to be related to the specialization of the various parts. Braille patterns, for instance, are typically touched with the palmar surface of the finger tips. Surface temperature, on the other hand, is often estimated using the dorsal side of the hand. In haptic perception of shape, often the whole hand is used. However, due to the inhomogeneity of the hand, it is conceivable that some parts are more sensitive to certain kinds of shape information (e.g., curvature) than others.

As a result of measurements of two-point thresholds, pressure sensitivity, and point localization thresholds, it is known that the spatial resolution of tactile stimuli var-

The authors are grateful to the Netherlands Organization for Scientific Research (NWO) for funding this project. We thank Susan te Pas, Irene Hoitink, and Ronald Burger for acting as observers, and Sheila $\mathrm{McNab}$, whose critical comments have improved the language style of the manuscript. Special thanks go to Hans Kolijn and Pieter Schiphorst for their technical help. Correspondence should be addressed to S. C. Pont, Rapenburg 77, 1011 TV Amsterdam, The Netherlands. ies over the body and even over the hand itself. For example, the sensitivity is much lower on the palm than on the fingertips of the hand, and it is also lower on the dorsal than on the palmar side of the hand (Loomis \& Lederman, 1986; Weber, 1834/1978; Weinstein, 1968). The two-point thresholds are correlated with the innervation density of the cutaneous type I mechanoreceptors of the skin of the hand (Vallbo, 1996; Vallbo \& Johansson, 1978). LaMotte and Srinivasan (1993) and Johnson, Hsiao, and Twombly (1996) also found evidence that the slowly adapting type I transduction mechanisms govern tactile form recognition. However, it has not yet been determined whether the sensitivity for shape perception also covaries with the two-point thresholds for different loci on the hand. In this paper, we want to map the curvature discrimination performance of different parts of the hand. Such knowledge is necessary if we want to understand how stimulations of separate regions of the hand are combined in 3-D shape perception.

Kappers, Koenderink, and te Pas (1994) investigated active haptic discrimination of doubly curved solid objects (quadric surfaces) in a quantitative way. They found that shape did not influence the active haptic discrimination of these hand-sized objects when the overall curvature was kept constant. We decided to investigate the discrimination of surfaces that are smaller and cylindrically curved because we wanted to distinguish among several different parts of the hand. The surfaces were touched statically in the present investigation because the position of the stimuli on the hand had to be varied systematically and precisely. Although it seems more typical to explore objects dynamically (Davidson, 1972; Lederman \& Klatzky, 1987), subjects are very well able to determine the curvature of objects when exploratory strategies are restricted (Davidson \& Whitson, 1974). Also, Lederman and Klatzky found that subjects typically used an ex- 
ploratory procedure involving static touch ("enclosure") to judge the global shape of objects. Moreover, the accuracy of subjects' judgments of global shape was highest when they were restricted to an exploratory procedure that they call "static contact." Thus, the use of static touch is not a limiting factor for investigating whether sensitivity for curvature discrimination varies over the hand.

Discrimination of curved stimuli has been investigated only for very specific situations. Rubin (1936), Crewdson and Zangwill (1940), and Hunter (1954) reported on the perception of straightness of edges when these were actively touched with the fingertips. They found that an edge may be perceived as straight when it is curved away from the subjects. Vogels, Kappers, and Koenderink (1996) also showed that haptic categorization (convexness or concaveness) of curvature is often not veridical.

Davidson (1972) investigated the haptic judgments that blind and sighted subjects made about curved strips with a length of $20 \mathrm{~cm}$ that they touched actively. His study concerned judgments of concaveness, convexness, or straightness of strips with curvatures ranging from $-1.6 / \mathrm{m}$ to $+1.6 / \mathrm{m}$. He concluded that scanning technique determines the veridicality and reproducibility of the judgments. Davidson and Whitson (1974) tested haptic matching of curvature. The stimuli were the same as in the previous investigation. Again, they showed how performance depends on exploratory strategies, but not how it depends on curvature. Thus it is not possible to determine discrimination thresholds from these studies.

Kappers and Koenderink (1996) showed that active haptic discrimination of cylindrically curved, hand-sized surfaces does not follow Weber's law; performance was relatively better with larger absolute curvatures. Gordon and Morison (1982) investigated the discrimination and the rating of curvature of much smaller stimuli that were actively touched with the index finger. These stimuli had a width of $10 \mathrm{~mm}$, a length of at least 10 and at most $40 \mathrm{~mm}$, and a curvature in the range of $0 / \mathrm{m}$ to $3 / \mathrm{m}$. They found that the effective stimulus for curvature is represented by the first-order geometrical structure - that is, the total attitude change over the surface of a curved stimulus, which is proportional to the base-to-peak height divided by half the length of the stimulus. So, if we compare stimuli that have the same curvature but are of different lengths, the longest stinıulus has the largest total attitude change over the surface and thus results in better curvature discrimination. The threshold for discrimination of a curved stimulus from a flat surface for the longest $(40 \mathrm{~mm})$ stimuli was $0.9 / \mathrm{m}$.

Goodwin, John, and Marceglia (1991) measured curvature discrimination of lenticular surfaces that were passively touched with a single fingerpad. The stimuli with a diameter of about $1 \mathrm{~cm}$ were pushed onto the fingerpad of the immobilized index finger. Goodwin et al. found that surfaces with curvatures of $+4.9 / \mathrm{m}$ and $-5.4 / \mathrm{m}$ can be discriminated from a flat surface. Performance was slightly better for larger curvatures.
Here we report on the static haptic discrimination of curved strips placed on different parts of the hand. The maximum curvatures of the stimuli we used in the present investigation were $-1.8 / \mathrm{m}$ and $+1.8 / \mathrm{m}$ and thus cannot be distinguished from a flat surface using only cutaneous stimulation from a single fingerpad (Goodwin et al., 1991). Thus, to judge the curvatures of these objects, it is necessary to combine stimulations - cutaneous, kinesthetic, or both-from different regions of contact with the stimuli.

\section{The Present Study}

Our aims in the present study were threefold. First, we wanted to map the sensitivity for discrimination of curvature for the different structures of the hand. In Experiment 1 , we measured discrimination thresholds for three different reference curvatures and nine different placements on the hand. To enforce the desired positions on the hand, the stimuli were touched statically instead of dynamically.

Second, we wanted to know which kind of stimulation-kinesthetic or cutaneous-is the more important in the discrimination of curved strips. When curved strips are touched with the dorsal side of the hand, the spatial resolution of the cutaneous stimulation will be poorer, whereas the resolution of kinesthetic stimulation will be the same as when the strips are touched with the palmar side of the hand. In Experiment 1, we also measured the curvature discrimination thresholds for the dorsal side of the hand and compared these to the results for the palmar side. Consequently, we were able to draw some conclusions about the relative importance of cutaneous stimulation in this task.

Third, we wanted to investigate whether the sensitivity for curvature discrimination correlates primarily with structural properties of the hand and/or with geometrical properties of the stimulus. The thresholds for curvature discrimination could, for instance, be correlated with the innervation density of the cutaneous receptors, as is the case for two-point thresholds. The number of joints involved is another variable that should be considered. But the curvature discrimination thresholds could also be correlated with the zeroth-, first-, or second-order geometrical properties of the stimulus, which are represented by the base-to-peak height difference, the total change of local surface attitude, and the curvature, respectively. In Experiment 2, we measured discrimination thresholds for different lengths and placements of the stimuli. We then further analyzed the results of Experiments 1 and 2 .

\section{EXPERIMENT 1}

This experiment examined whether the sensitivity for curvature discrimination varies over the palmar side of the hand. This was done by means of measurements of the discrimination thresholds for nine different placements on the hand and three reference curvatures. The 

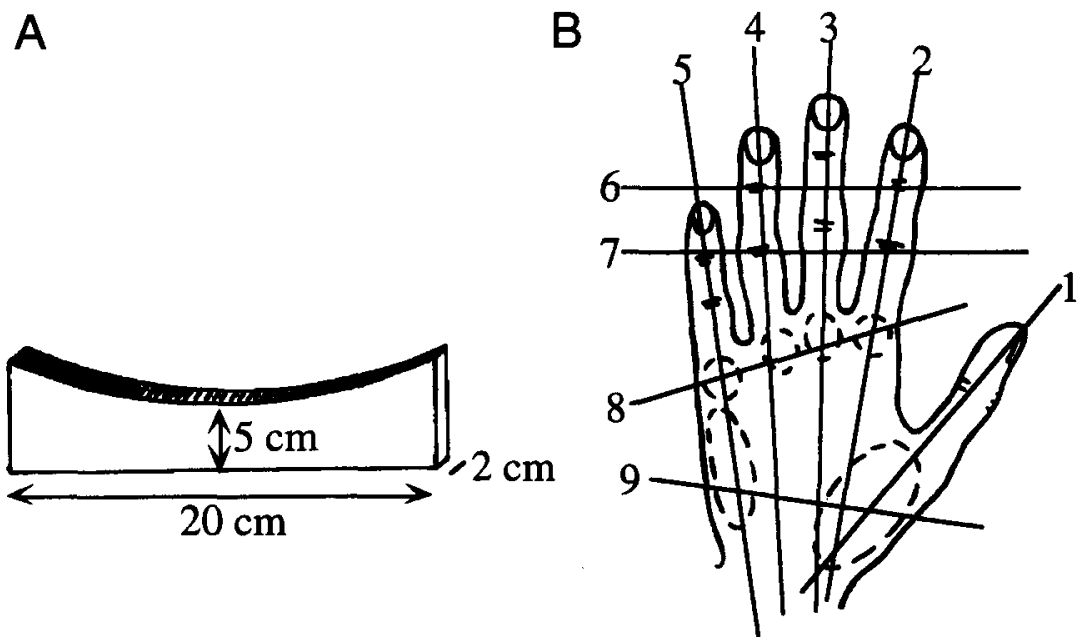

Figure 1. (A) A concave (negatively curved) strip. For clarity the strip has been depicted with a much larger curvature than the strips actually had. (B) The nine different placements on the hand. The strips were $20 \mathrm{~cm}$ long, $2 \mathrm{~cm}$ wide, and $5 \mathrm{~cm}$ high in the middle of the strip. In placements 1-5, the strips were placed along the first to fifth digits plus the areas of the palm of the hand that are along the same lines as these digits. In placements 6-9, the strips are placed perpendicular to the fingers. In placements $6-7$, the strips were placed on the distal and proximal joints, respectively, of the second (index) to fourth (ring) fingers. The strips were placed on the knuckles in placement 8 and on the paim, over the thenar eminence, in placement 9.

relative importance of cutaneous and kinesthetic stimulation in this task was investigated qualitatively by means of comparison of the discrimination thresholds in a palmar and a dorsal condition. We expected to find large differences between the thresholds for the different placements due to the inhomogeneity of the hand. For instance, we expected much worse performance for the placement in which the strips were touched primarily with the thenar eminence than for the one in which they were touched with the distal parts of the fingers. The innervation density of the cutaneous mechanoreceptors is lower at the first placement, and no joints are involved.

\section{Method}

Subjects. Three subjects, 1 male (R.B.) and 2 female (I.H. and S.P.), participated in the experiments. Subjects R.B. and I.H. were naive and paid for their efforts. S.P. (not one of the authors) was experienced in haptic experiments and participated on a voluntary basis. All subjects were strongly right-handed according to the definition used by Coren (1993).

Stimuli. We have investigated the discrimination of three reference curvatures (a concave, convex, or straight strip) from several other curved strips. The stimuli were circular arcs with a length of $20 \mathrm{~cm}$, a width of $2 \mathrm{~cm}$, and curvatures between $-1.8 / \mathrm{m}$ and $+1.8 / \mathrm{m}$. The curvature is the reciprocal of the radius of curvature and vice versa, so a curvature of $k=1.8 / \mathrm{m}$ is represented by a circle with a radius of $(1 / 1.8) \mathrm{m}=0.56 \mathrm{~m}$. Figure $1 \mathrm{~A}$ shows an example of a negatively curved (concave) strip. For clarity, it has been depicted with a much larger curvature than the strips actually had. The concave, straight, and convex reference strips had curvatures of $-0.8 / \mathrm{m}, 0 / \mathrm{m}$, and $+0.8 / \mathrm{m}$, respectively. These stimuli were combined with strips the curvatures of which differed from those of the reference strips by $-1.0 / \mathrm{m}$ to $+1.0 / \mathrm{m}$, with a constant stepwidth of $0.2 / \mathrm{m}$.
Experimental setup. The subjects were seated behind a curtain that prevented them from seeing the experimenter and the stimuli. We chose to use a curtain instead of blindfolding the subjects because they were more comfortable with this setup in the long term (the experiments described in the present paper consisted of 45 sessions of about an hour for R.B. and I.H., and 8 sessions for S.P.). Moreover, this setup enabled the subjects to write down their judgments themselves, so that the experimenter had more time to prepare the next stimulus presentation. As a result, a rate of presentation of about 192 trials in $30 \mathrm{~min}$ was possible. This rate was comfortable for the subjects, who were not limited in the time they (statically) touched the stimuli but who did not need much time to make their judgments (about $9.5 \mathrm{sec}$ were needed for each trial, which consisted of two presentations of strips).

The subject put his/her right hand under the curtain to touch the stimuli presented by the experimenter. The subject rested his/her elbow on the table and moved his/her relaxed hand up and down in the same place during all sessions. The subject's hand moved onto the stimulus to touch it in a static manner. The strips were fixed in a frame so that they were always presented in the same place during a given condition. In this way, we enforced the position of the hand on the stimulus, which was checked by the experimenter. To vary the place of presentation of the stimuli in between conditions, the frame was moved into the other desired positions.

The frame was turned upside-down when the strips were to be presented in the dorsal condition. Thus the shapes were presented upright under the hand in the palmar condition and upside-down above the hand in the dorsal condition. We tested the dorsal condition with this experimental arrangement to make sure that the kinesthetic stimulation was similar in the palmar and dorsal conditions. As a result, it was possible to determine whether stimulation of the skin is important in haptic curvature discrimination.

Procedure. Because curvature perception is not veridical (Crewdson \& Zangwill, 1940; Hunter, 1954; Rubin, 1936; Vogels et al., 1996), we chose to investigate the sensitivity for curvature by means of a discrimination task instead of using absolute judg- 


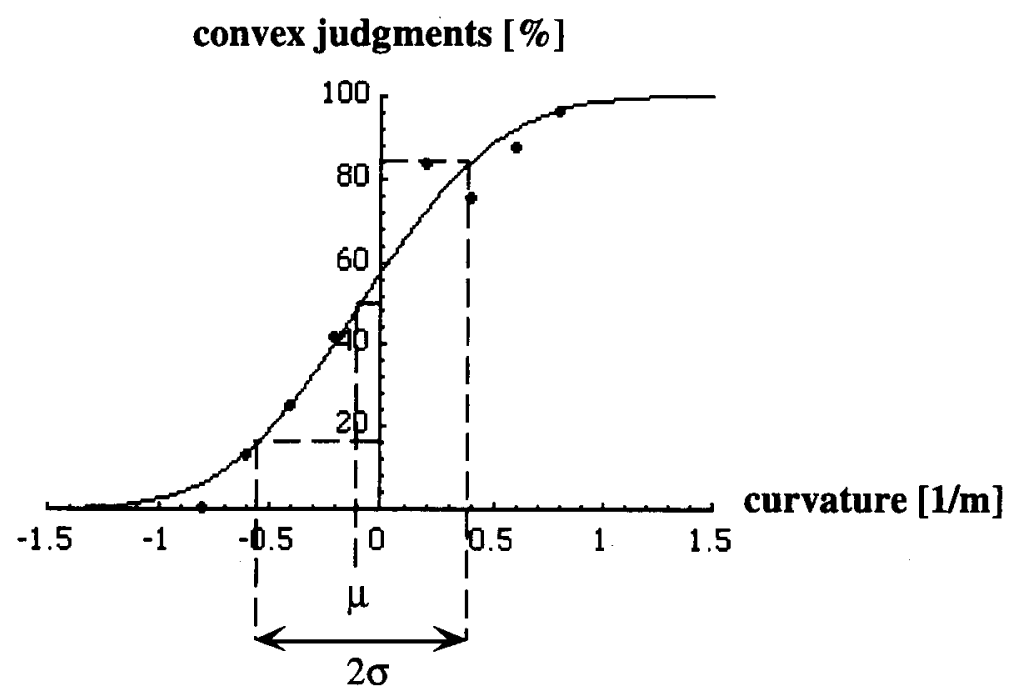

Figure 2. A representative example of data points and the psychometric function that was fitted to these data points. The vertical axis shows the percentages of judgments in which the test shapes were judged more convex than the reference shape. The horizontal axis shows the differences between the curvatures of the test strips and the reference strip. Sigma, or the discrimination threshold at $84 \%$ correct, is the difference between the $84 \%$ and the $50 \%$ points, which is inversely related to the steepness of the curve. Mu is the $50 \%$ point or shift.

ments. In such a task, variations in curvature perception, such as shifts in the phenomenal flatness (Vogels et al., 1996), cannot influence the measurements for this sensitivity. Moreover, discrimination of curved strips from curved reference strips can also be tested with this procedure. We used a two-alternative forced choice procedure. Using static touch, subjects had to judge which of two successively presented stimuli was the more positively curved.

This experiment was done for nine different placements of the stimuli on the hand. Because of the heterogeneous structure of the hand, we expected the sensitivity for curvature discrimination to vary with placement. The nine different placements are shown in Figure $1 \mathrm{~B}$. In placements $1-5$, the strips were placed along the first to fifth digits plus the areas of the palm of the hand that were along the same lines as these fingers. In placements 6-9, the strips were placed perpendicular to the fingers. In placements $6-7$, the strips were placed on the distal and proximal joints, respectively, of the second to fourth digits (index, middle, and ring fingers). In placement 8 , the strips were placed on the knuckles and in placement 9 on the palm, over the thenar eminence. The palmar and the dorsal conditions were tested for all nine placements.

To check for effects of the experimental setup, gravity, additional lifting, applied force, and the position of the stimulus in space, we set up two control conditions: for placements 3 and 9 , the dorsal and palmar condition were measured with the hand turned palm upward (so the stimulus was fixed upright under the hand in the dorsal condition and upside-down above the hand in the palmar condition).

In each measurement with Subjects R.B. and I.H., the test strips differed $\pm 0.4 / \mathrm{m}, \pm 0.6 / \mathrm{m}, \pm 0.8 / \mathrm{m}$, and $\pm 1.0 / \mathrm{m}$, or $\pm 0.2 / \mathrm{m}$, $\pm 0.4 / \mathrm{m}, \pm 0.6 / \mathrm{m}$, and $\pm 0.8 / \mathrm{m}$ from the reference strips, depending on the exact position, condition, and performance in earlier measurements. The test curvatures were spaced evenly around the reference curvature. All combinations were presented four times in one order - first the reference and then the test strip and four times in the other order-first the test and then the reference strip-in a random sequence. Each experimental condition thus consisted of 3 (reference shapes) $\times 8$ (combination shapes) $\times 4$ (trials) $\times 2$ (orders) $=192$ trials and was tested three times on dif- ferent days. In the measurements with Subject S.P., we tested only 10 different combinations of the reference strips $(-0.8 / \mathrm{m}, 0 / \mathrm{m}$, and $+0.8 / \mathrm{m}$ ) with strips with a difference of $\pm 0.4 / \mathrm{m}$ or $\pm 0.8 / \mathrm{m}$. All combinations were presented five times in one order and five times in the other order in a random way. Each experiment with Subject S.P. thus consisted of 10 (different combinations) $\times 5$ (trials) $\times 2$ (orders) $=100$ trials. In sum, there were three reference curvatures, which were combined with eight test curvatures each for R.B. and I.H., but four test curvatures each for S.P. The test curvatures differed depending on conditions, and several were deleted for S.P.

Subjects I.H. and R.B. thus judged 11 (placements) $\times 2$ (palmar and dorsal condition) $\times 3$ (measurements) $\times 192$ (trials) $=$ 12,672 combinations of two strips. Subject S.P. judged $11 \times 2 \times$ $100=2,200$ combinations of two strips. In about $30 \mathrm{~min}, 192$ trials were measured, so this experiment took about $72 \mathrm{~h}$ of measurements. With each subject, we measured for about $1 \mathrm{~h}$ each session, testing the palmar and dorsal conditions for one placement. The palmar and dorsal conditions were measured separately. This was necessary because of the practical constraint that the frame had to be turned upside-down and fixed in the correct position in between these experiments. The order in which the experimental conditions were tested was different for each subject and for each set of measurements.

Analysis. Psychometric curves (cumulative Gaussian distributions) were determined for the percentages of judgments in which the test shapes were judged more convex than the reference shape as a function of the differences between the curvatures of the test strips and the reference strip. Because the range of test curvature varied from session to session, the data points were weighted linearly with the numbers of trials of which these percentages of judgments consisted, because the variances of these points are, theoretically, inversely related to these numbers. The LevenbergMarquardt method (Press, Flannery, Teukolsky, \& Vetterling, 1988) was used to fit the curves. Figure 2 shows a representative example of how the curves fit the data. A psychometric curve can be characterized by its location $(\mu)$ and its slope $(\sigma)$. Mu (the point of subjective equality) represents the $50 \%$ point or the mean 


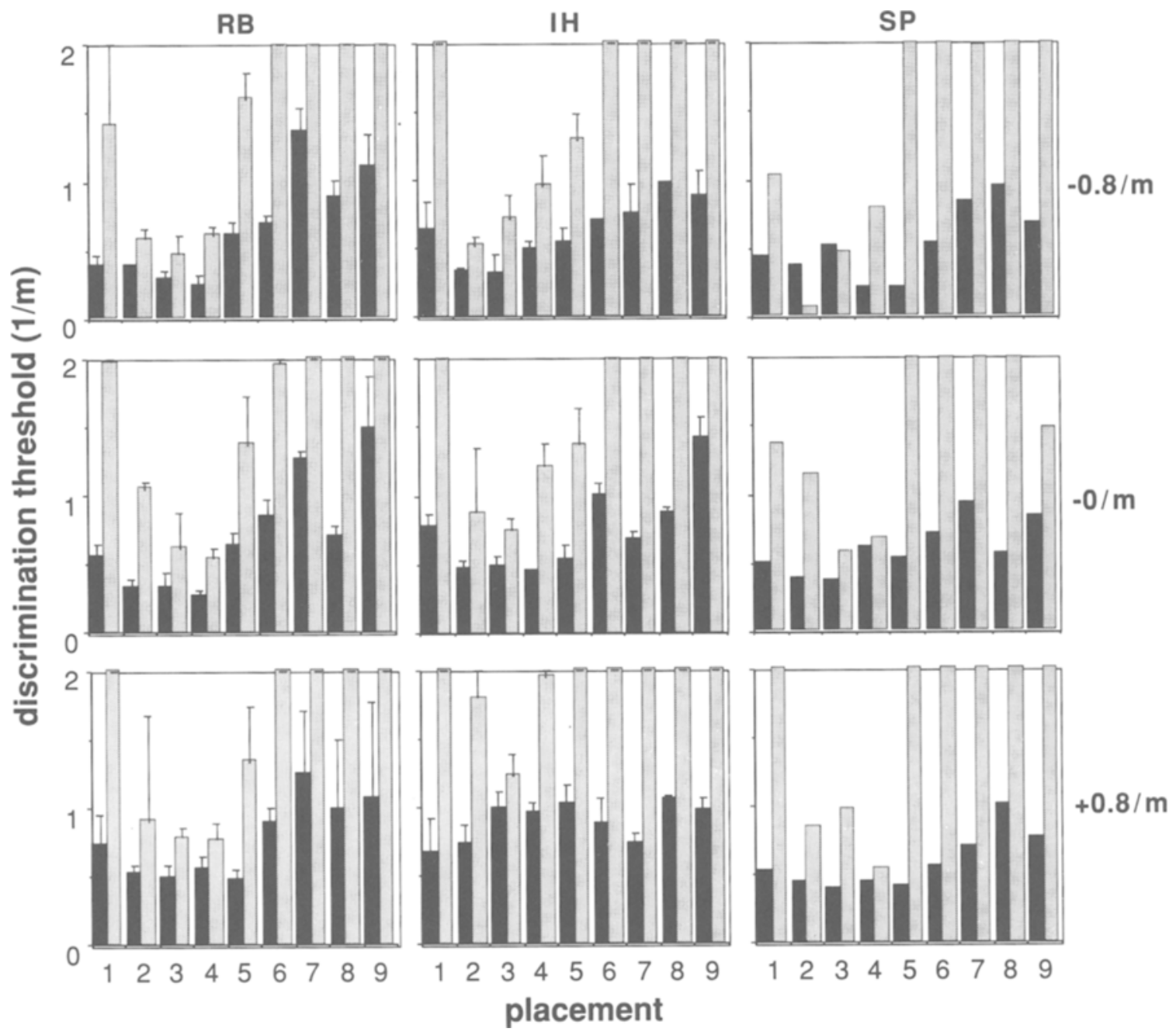

Figure 3. Discrimination thresholds expressed in units of curvature for Subjects R.B. (left panels), I.H. (middle panels), and S.P. (right panels). The thresholds are shown for all three reference curvatures $(-0.8 / \mathrm{m}, 0 / \mathrm{m}$, and $+0.8 / \mathrm{m})$ in the upper, middle, and lower panels, respectively. The numbers that correspond to the nine different placements in Figure 1 are shown on the horizontal axis. The black and gray bars show the values for the thresholds in the palmar and dorsal conditions, respectively. The error bars represent the standard errors, which are determined over the three repeated measurements.

(Macmillan \& Creelman, 1991). This value should not deviate too much from the reference value. Sigma is the most interesting parameter. It is inversely related to the steepness of the curve and represents the discrimination threshold at $84 \%$ correct (Macmillan \& Creelman, 1991). To give an indication of the errors in these values, we calculated standard errors over the parameters of separate curves for each of the three measurements per condition.

\section{Results}

The means of the psychometric curves did not differ systematically from the reference values, as expected. In the case of our discrimination task, influences of biases were counterbalanced and thus there should have been no difference between the reference curvature and the curvature that was subjectively equal to it.

Figure 3 shows the discrimination thresholds for Subjects R.B. (left panels), I.H. (middle panels), and S.P. (right panels). The results for R.B. and I.H. are shown with standard errors, which were calculated over the pa- rameters for the three measurements per condition. S.P. measured each condition once and thus we could not determine errors for her results. It is reasonable to assume that the errors in these data are at least as large as those in the results for R.B. and I.H., and probably somewhat larger (because S.P.'s thresholds are based on many fewer trials). The nine different placements are represented on the horizontal axis. The numbers correspond to those in Figure 1. The discrimination thresholds are given on the vertical axis in units of curvature $(1 / \mathrm{m})$. The black and gray bars represent the thresholds, which were measured in the palmar and dorsal conditions, respectively. Thresholds higher than $2 / \mathrm{m}$ are not specified because these values exceed the experimental range and are thus illdefined. This is the case for some values for the dorsal condition. We did not test these cases more accurately because it is already clear from these data that the sensitivity for curvature was very low in these conditions. The 
Table 1

Ranges and Mean Values of the Discrimination

Thresholds Expressed in Units of Curvature for the Two

Clusters of Placements: Along the Fingers (Placements 1-5)

and Perpendicular to the Fingers (Placements 6-9).

\begin{tabular}{ccccccc}
\hline & & \multicolumn{3}{c}{ Discrimination Thresholds $(1 / \mathrm{m})$} \\
\cline { 3 - 4 } Subject & $\begin{array}{c}\text { Reference } \\
\text { Curvature }[1 / \mathrm{m}]\end{array}$ & \multicolumn{2}{c}{ Placements $1-5$} & & \multicolumn{2}{c}{ Placements 6-9 } \\
\cline { 3 - 4 } \cline { 6 - 7 } & Range & Mean & & Range & Mean \\
\hline R.B. & -0.8 & $.24-0.61$ & .38 & & $.70-1.38$ & 1.02 \\
& 0 & $.27-0.64$ & .43 & & $.71-1.48$ & 1.07 \\
I.H. & +0.8 & $.48-0.73$ & .56 & & $.89-1.26$ & 1.05 \\
& -0.8 & $.32-0.63$ & .46 & & $.70-0.97$ & 0.83 \\
& 0 & $.46-0.79$ & .55 & & $.69-1.43$ & 1.00 \\
S.P. & +0.8 & $.67-1.02$ & .88 & & $.74-1.05$ & 0.91 \\
& -0.8 & $.22-0.52$ & .35 & & $.54-0.96$ & 0.75 \\
& 0 & $.37-0.61$ & .48 & & $.57-0.93$ & 0.77 \\
& +0.8 & $.40-0.52$ & .45 & & $.55-1.01$ & 0.76 \\
\hline
\end{tabular}

Note-The values are given for all three reference curvatures and all 3 subjects.

discrimination thresholds for the palmar condition were always lower than $2 / \mathrm{m}$ and thus well defined.

The upper, middle, and lower panels of Figure 3 show the thresholds in the case of discrimination of strips from a reference strip with a curvature of $-0.8 / \mathrm{m}, 0 / \mathrm{m}$, and $+0.8 / \mathrm{m}$, respectively. To investigate whether reference curvature and position had significant effects on the discrimination thresholds, we performed a 2-way related analysis of variance (ANOVA) on the results for the palmar conditions. The results showed that reference curvature had no significant effect on the thresholds $[F(2,4)=$ $4.60, p>.05]$.

Position, however, did have a significant effect $[F(8,16)$ $=7.51, p<.001]$. Overall, the sensitivities for curvature discrimination for placements $1-5$ were higher than those for placements 6-9. The ranges and the mean values for the thresholds for these two clusters are given for all 3 subjects in Table 1. The thresholds for placements $1-5$ were approximately $0.5 / \mathrm{m}$, and those for placements $6-9$ were approximately $0.9 / \mathrm{m}$. So, the sensitivity for curvature discrimination was about twice as low when the strips were touched perpendicular to the fingers than when they were touched along the fingers.

Finally, there was a significant interaction of reference curvature $\times$ position $[F(16,32)=2.39, p<.025]$. Table 1 shows that the thresholds for placements along the fingers were $h$ igher for the convex reference curvature. For placements perpendicular to the fingers, thresholds were constant in this range.

In Figure 3, the gray bars are higher than the black bars for almost all conditions, indicating that discrimination was poorer in the dorsal than in the palmar condition. Figure 4 shows the discrimination thresholds for the control conditions, together with the results for the corresponding placements in the palm-down conditions (also shown in Figure 3 ). The manner of presentation in Figure 4 is analogous to that in Figure 3. The thresholds in placements 3 and 9 in both the palmar and dorsal conditions were, within the errors, equal for the palm-down and the palm-upward (control) conditions. So, the reduction in performance in the dorsal condition indicated by
Figure 3 cannot have been caused by the experimental setup. Thus, although the kinesthetic stimulation of the hand was the same overall, the sensitivity for curvature discrimination was lower for the dorsal than for the palmar condition, on a corresponding placement. This shows that the stimulation of the skin does play an important role in the discrimination of curved strips in the range of $-1.8 / \mathrm{m}$ to $+1.8 / \mathrm{m}$.

\section{Discussion}

Discrimination along the fingers is better than discrimination perpendicular to the fingers. The larger contact area in the first case might explain this. Another possibility is that it may be less difficult to combine (cutaneous and kinesthetic) stimulations along one finger than over several fingers, because the involved phalanxes are joined in the first case and not in the second.

To compare the results in the palmar and dorsal conditions, we assumed that the kinesthetic stimulation was similar in these cases. Possible effects of postural differences were presumed to be small because the surfaces that were used were relatively "flat" (the largest height difference was $9 \mathrm{~mm}$ over a length of $20 \mathrm{~cm}$ ). A difference between the palmar and dorsal conditions that resulted from our experimental setup was that in the palmar case, the hand was placed on the strip, whereas in the dorsal case, the hand had to be held actively against the strip, which was above the hand. This was reversed for the control conditions, in which the hand was turned palm upward. The thresholds, however, did not differ for the palm-down and palm-up conditions. As an extra verification, we compared the results for the palmar condition for reference curvatures of $-0.8 / \mathrm{m}$ and $+0.8 / \mathrm{m}$ with those for the dorsal condition for reference curvatures of $+0.8 / \mathrm{m}$ and $-0.8 / \mathrm{m}$, respectively. Thus, this comparison was made for hand postures that are almost the same instead of a comparison for similar stimuli. However, here we also found that performance was poorer for the dorsal than for the palmar condition. The difference between the dorsal and palmar conditions was not significant only for placements $2-4$. In sum, the reduction in 

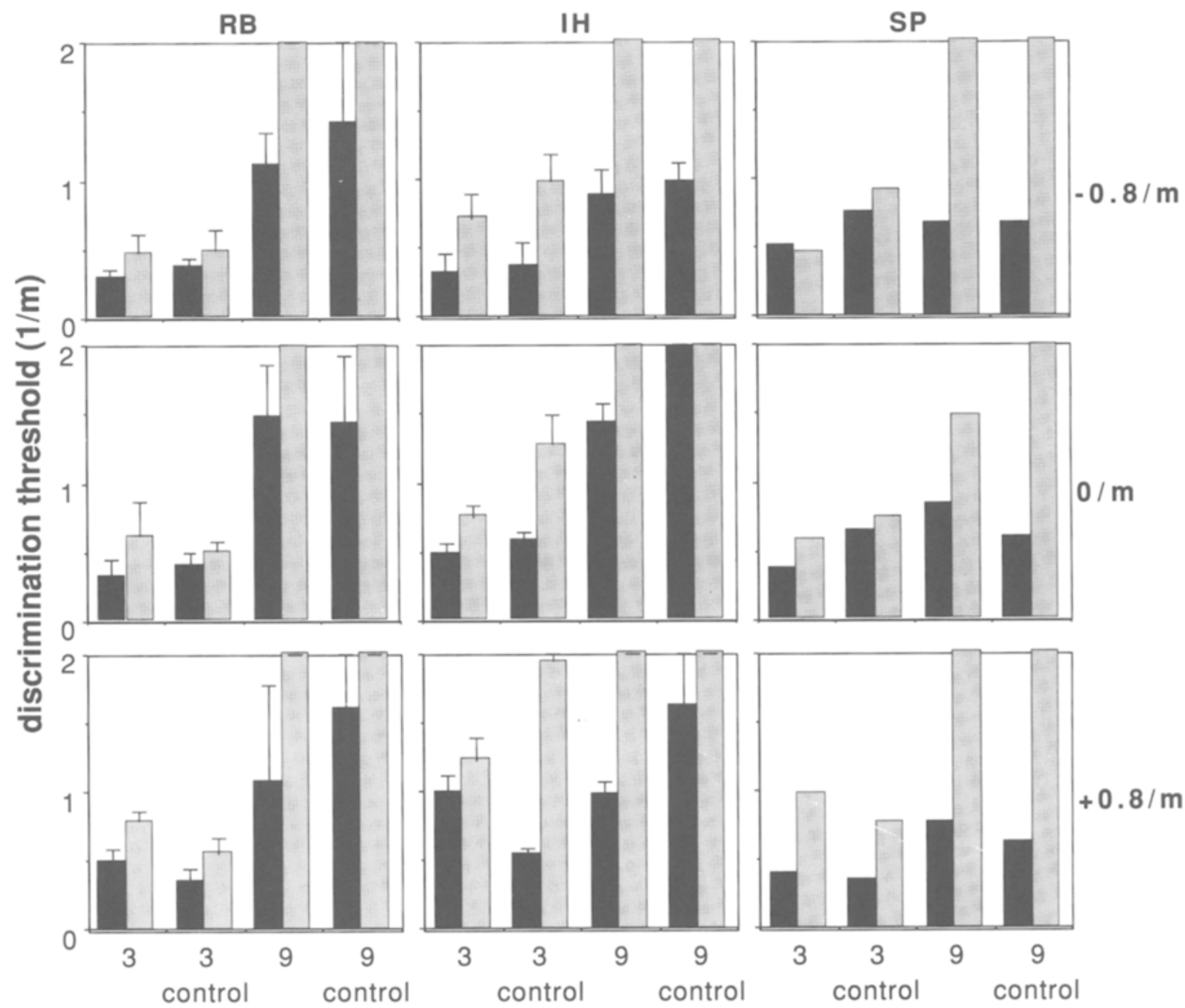

\section{placement}

Figure 4. Discrimination thresholds for the control experiments (placements 3 control and 9 control). The thresholds that were found for placements 3 and 9 are also shown for comparison. The data are represented analogously to those in Figure 3.

performance in the dorsal, compared with the palmar, condition cannot have been caused by the experimental setup but must be an effect of the lower cutaneous resolution on the dorsal side of the hand. So, cutaneous stimulation is important for the static discrimination of curved strips, especially when the strips are placed perpendicular to the fingers.

\section{EXPERIMENT 2}

Because the total length over which the strips were touched seemed to covary with the thresholds for the different placements, a second experiment was done to investigate whether differences in the discrimination thresholds were primarily due to the length of the stimulus area contacted. For this purpose, we tested discrimination for several different parts of the palmar hand for different lengths of the contact area, using shorter strips ( 8 instead of $20 \mathrm{~cm}$ in length). Also, for placement 6 , we tested two conditions in which the contact area lengths differed. This was done by means of other manners of positioning the fingers: The contact area was increased or shortened by spreading the fingers out wide or by holding the fingers close together, respectively. We expected to find large effects due to the structural properties of the hand, not due to the contact length.

\section{Method}

Subjects. The subjects who participated in Experiment 2 were the same as those who participated in Experiment 1.

Stimuli. In this experiment, we used only a straight strip as reference strip because in Experiment 1 the curvature of the reference strip was found to have no significant effect on discrimination thresholds. The straight reference strip was combined with eight different test curvatures in the range $-1.6 / \mathrm{m}$ to $+1.6 / \mathrm{m}$. In six of the eight conditions in this experiment, we used strips of 8 instead of $20 \mathrm{~cm}$ in length. In the two conditions for placement 6 , 

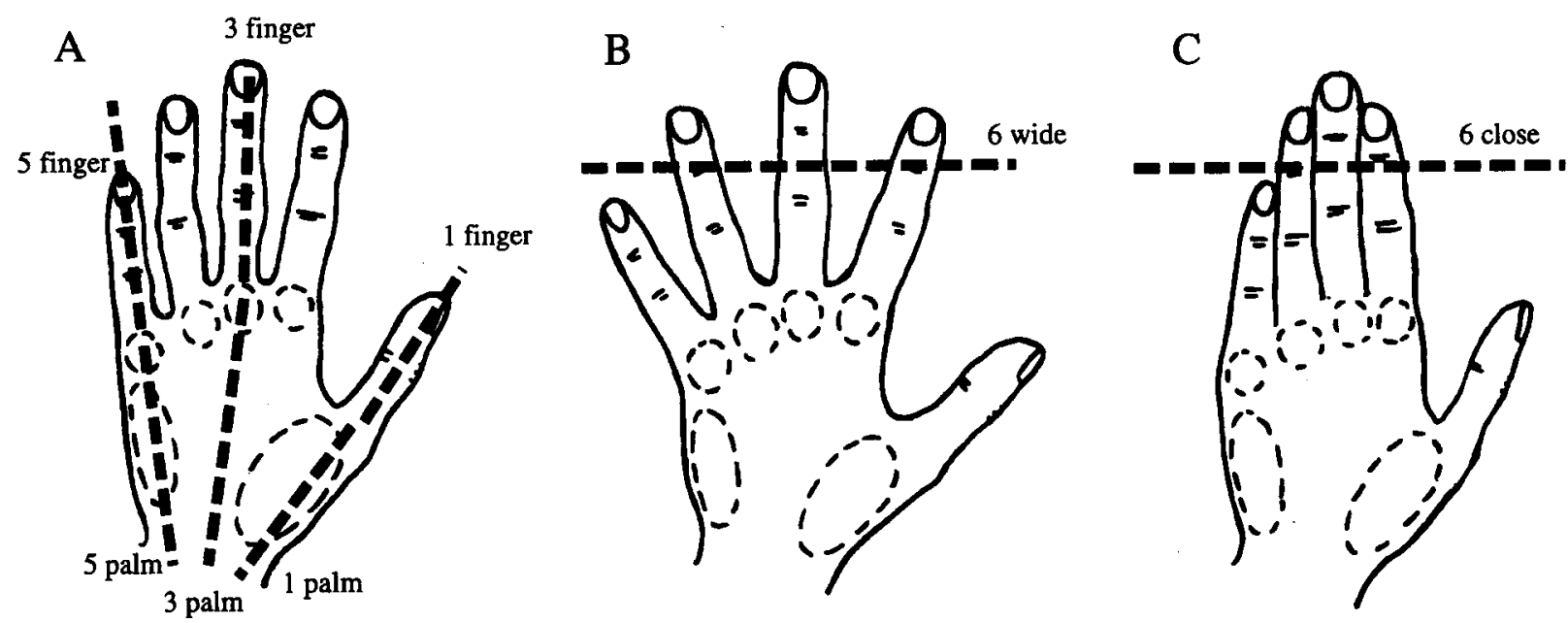

Figure 5. Schematic representation of the eight conditions that were tested in Experiment 2. (A) The conditions in which strips with a length of $8 \mathrm{~cm}$ were used. These were presented on the finger only in placements 1,3 , and 5 (1 finger, 3 finger, and 5 finger, respectively), or on the part of the palm that corresponds to these placements (1 palm, 3 palm, and 5 palm). (B) and (C) The conditions " 6 wide" and " 6 close," in which the strips were touched with the fingers spread out wide and held close together, respectively, on placement 6.

we used the $20-\mathrm{cm}$-long strips because in the condition in which the fingers were widely spread, the length over which the strips were touched exceeded $8 \mathrm{~cm}$.

Experimental setup. The experimental setup was the same as that in Experiment 1: The strips were fixed upright in a frame and were touched with the palmar side of the hand, which was turned palm down. The dorsal condition was not tested.

Procedure. Eight conditions were tested (Figure 5). In the case of the thumb (placement 1), middle finger (placement 3 ), and little finger (placement 5), two conditions were tested with 8- instead of 20-cm strips: In one condition, these shapes were touched with the finger only, and in the other condition, the shapes were touched with the part of the palm of the hand that is involved in these placements (Figure 5A). In placement 6 , one condition was tested in which the subject had to spread the fingers as much as possible (Figure 5B). For this placement, we also tested a condition in which the subject had to touch the strips with the fingers held together (Figure 5C). Thus the total length over which the strip was touched (length of contact) was larger ("wide" condition) or smaller ("close" condition) than in the previous experiment. The range in which discrimination was tested differed for the different conditions and subjects and was adjusted after each test on the basis of performance in the previous measurement. We again measured about 192 trials in $30 \mathrm{~min}$, so this experiment took about $26 \mathrm{~h}$ of measurement.

Analysis. The discrimination thresholds and standard errors were determined via the same procedures as those of Experiment 1 .

\section{Results}

The vertical axes in Figure 6 show the discrimination thresholds for R.B., I.H., and S.P. (upper, middle, and lower panels, respectively). The horizontal axis shows 12 conditions in four subsets of three conditions: eight conditions that were tested in this experiment and four conditions that had already been tested in Experiment 1 (1, 3 , and 5 "total" and 6 "normal"). The first, second, and third clusters show the results for the "finger-only," "palm-only," and "total" (finger and palm) conditions for the thumb, middle finger, and little finger, respectively. The contact length was in the same order of magnitude in the "finger" and "palm" conditions, whereas it was about twice as large in the "total" condition. Strikingly, the thresholds for the finger and palm conditions did not differ systematically. The threshold for the palm condition was (slightly) higher than for the finger condition in only 5 out of 12 cases. The performance for the total conditions was better than for the finger or palm conditions in 7 out of 12 cases and always better than for the palm conditions. Again, thus, the discrimination thresholds seemed to decrease for larger contact lengths. The fourth cluster depicts the results for three different contact lengths for placement 6 . Overall, the thresholds for this placement also decreased when contact length increased.

\section{Discussion}

We expected to find, for instance, that the palm conditions would give rise to higher discrimination thresholds than would the finger conditions. However, we did not find such systematic differences due to structural properties of the hand. It might be the case that the sensitivity for curvature discrimination correlates primarily with contact length - a geometrical property of the stimulus-instead of with the (anatomical and neurological) structure of the part of the hand with which the strips were touched. This hypothesis is tested in the following analysis. In addition, we try to describe the data in more detail by means of consideration of three mathematical models.

\section{FURTHER ANALYSIS OF THE RESULTS OF EXPERIMENTS 1 AND 2}

In order to further investigate the effect of contact length on discrimination performance, we looked at the data in relation to the geometrical properties of the stimuli instead of in relation to the different placements. The aim of the following analysis is to try to describe the data 

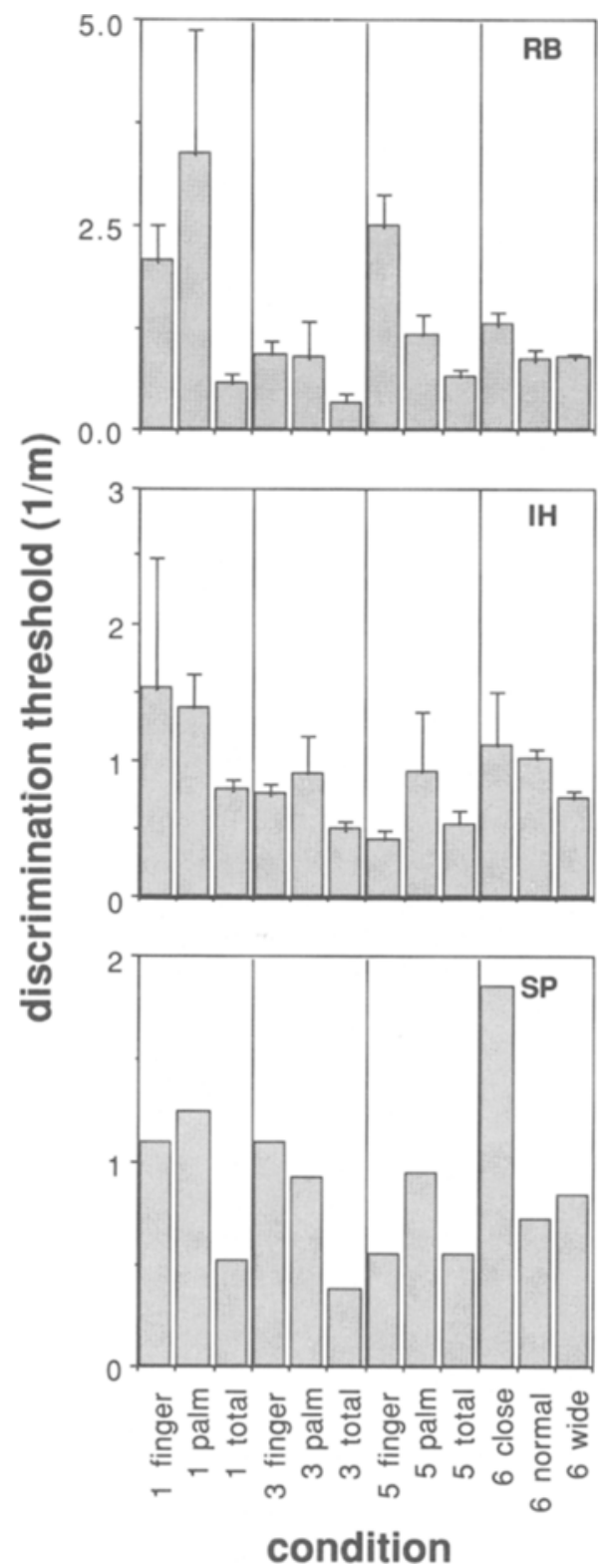

Figure 6. On the vertical axes in the upper, middle, and lower panels, the discrimination thresholds in terms of curvature are shown for R.B., I.H., and S.P., respectively. On the horizontal axes, 12 conditions are represented-eight placements that were tested in Experiment 2 and four conditions that had already been tested in Experiment 1.

presented in this paper in a simplified, meaningful way by means of a mathematical model. It should be noted that the three models considered here should be seen as possibilities for such a description, not as laws of nature that need verification. These three models represent limit cases for the discrimination of curved strips: discrimination on the basis of the zeroth-, first-, or secondorder geometrical structure (height differences, attitude differences, and curvature). The purpose of this analysis is to test whether the data-for all the conditions and contact lengths - conform to a single trend, and if they do, whether one of these limit cases can describe that trend.

Figure $7 \mathrm{~A}$ is a schematic representation of a hand touching a strip. A situation is depicted in which there are three fingers contacting a convex strip. The three fingers and the convexness are arbitrary choices; we could also have depicted, for instance, the four knuckles contacting a concave strip. The geometrical variables radius of curvature, local attitude, height difference, and contact length are clarified in this figure. Two different measures for contact length are used in the analysis-the centerto-center contact length and the total contact length. These measures are clarified in Figure 7B. The centerto-center contact length is the distance between the centers of the outermost areas that would touch the strips in a certain placement. The total contact length is the distance between the outermost spots that would contact the strips in a certain placement.

\section{The Three Models}

Description. In this section, we describe three models by which curvature discrimination might take place and compare the data of Experiments 1 and 2 to the predictions that follow from these models. The three models are defined by a constant threshold in terms of curvature, local surface attitude, or base-to-peak height differenceor, in other words, invariance of the second-, first-, or zeroth-order geometrical structure, respectively.

The three models are represented schematically in Figures $8 \mathrm{~A}-8 \mathrm{C}$. For clarity, the strips are depicted with a much larger curvature (which is equal to the reciprocal of the radius of curvature) than they actually had. For the curvatures we actually used, the length of the base of the arc and that of the arc differed at most by $1 \%$. The left panels in Figure 8 represent situations for shorter contact lengths, and the right panels those for longer contact lengths. The predictions that follow from the three models will become obvious through comparison of the first (left) situation and the second (right) situation.

For the discrimination of strips of constant curvature, one can think of several ways of processing the geometrical structure. The simplest way in which the discrimination can take place is to compare curvatures directly. If curvatures are compared directly, the sensitivity for curvature is expected to be determined by a constant threshold in terms of curvature. This model is represented schematically in Figure 8A. In this model or limit case, a strip with a certain (constant) radius of curvature, $R$, can just be discriminated from a straight strip, independent of the contact length. Thus, when the strips are touched over a length $2 x_{1}$ (left panel), a strip with a radius of curvature of $R$ can just be discriminated from a straight strip. Then the base-to-peak height difference over the contacted area is $y_{1}$. Note that only the height difference over the contacted area is taken into account, not the height difference over the whole stimulus. When the strips are touched over another length $2 x_{2}$ (right panel) that is larger than $2 x_{1}$, the radius of curvature that can 
A
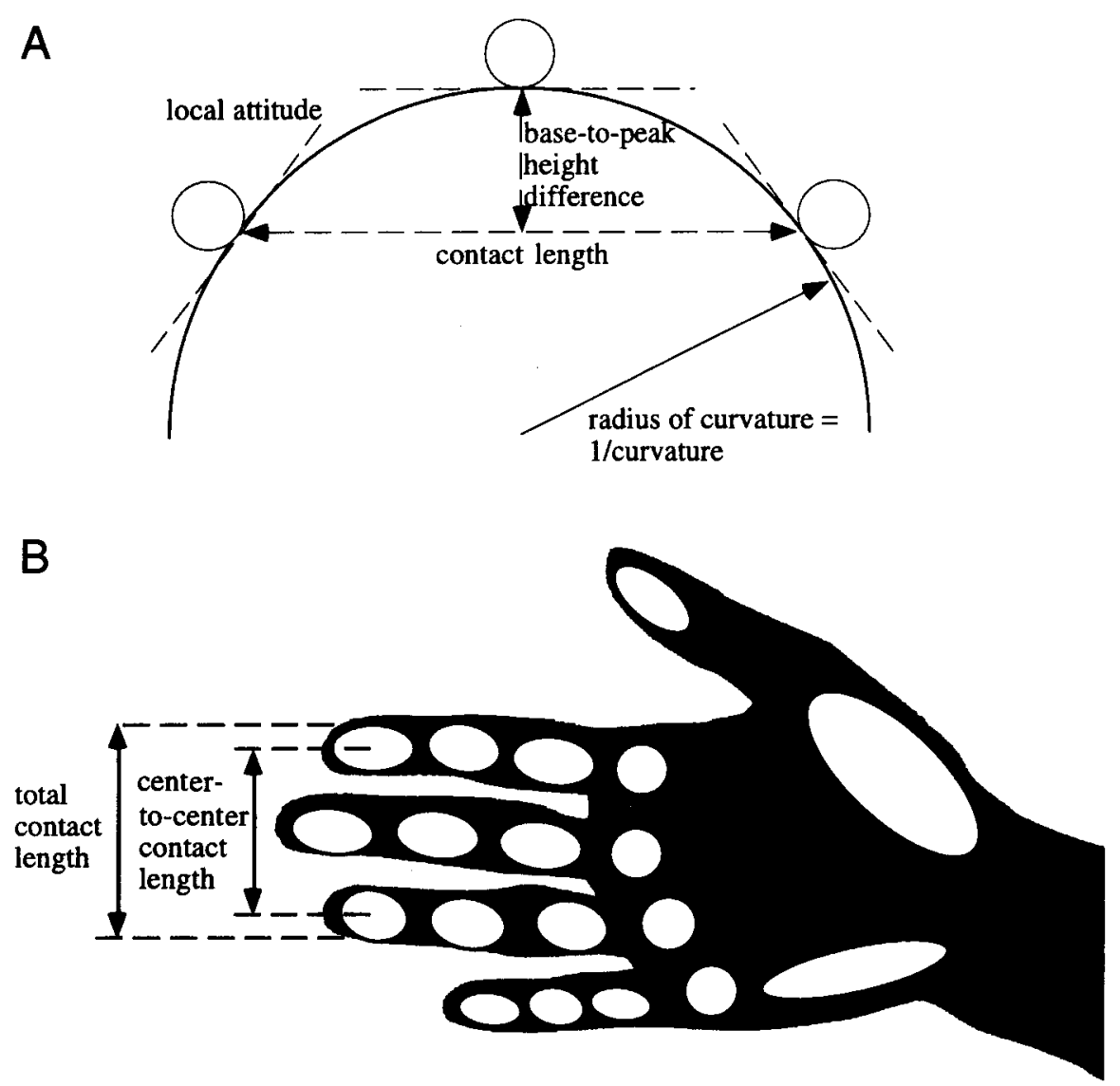

Figure 7. (A) A schematic representation of a hand touching a strip and (B) an example of a photocopy of a hand. Panel $A$ depicts a situation in which three fingers are contacting a convex strip. The three fingers and the convexness are arbitrary choices; we could also have depicted, for instance, the four knuckles contacting a concave strip. These figures serve only to clarify the different geometrical variables we used in our analysis - the radius of curvature, the local attitude, the height difference, and the contact length. Two different measures for contact length were used in the analysis - the center-to-center contact length and the total contact length. These measures are clarified in panel B. The center-to-center contact length is the distance between the centers of the outermost areas that touched the strips in a certain placement. The total contact length is the distance between the outermost spots that contacted the strips in a certain placement.

just be discriminated is still $R$. The height difference over the contacted area in that situation, $y_{2}$, is larger than $y_{1}$. The predictions for the thresholds expressed in units of radius of curvature and in units of height difference, as a function of contact length, are shown in Figure 9A (the mathematical derivation of these functions can be found in the Appendix). The threshold in terms of radius of curvature is a constant (the starting point in this case). The thresholds in terms of height difference then increase as a function of length ( $y_{2}$ is larger than $\left.y_{1}\right)$.

Another relatively simple way to discriminate curved strips might be to compare the overall gradients or local attitude differences over the surface. This is shown in Figure $8 \mathrm{~B}$. In other words, the threshold in terms of the local surface attitude is a constant. When we compare the left and right panels of Figure $8 \mathrm{~B}$, it is clear that when the contact length $x_{2}$ is larger than $x_{1}$ while the attitude difference over the touched part of the strip is the same, the height difference $y_{2}$ is larger than $y_{1}$, and the radius of curvature $R_{2}$ is larger than $R_{1}$. Moreover, the contact lengths, the height differences, and the radii of curvature all differ by the same factor. This means that when the length of the area over which the strip is contacted is larger by a ratio $x_{2} / x_{1}$, the threshold in terms of radius of curvature, as well as the threshold in terms of height difference, will also be larger by a factor of $x_{2} / x_{1}$. Thus, the situation in the right panel in Figure $8 \mathrm{~B}$ is a scaled (ratio $x_{2} / x_{1}$ ) version of that in the left panel. That is, discrimination of curved strips is scale invariant. According to this model, the thresholds in terms of radius of curvature and in terms of height difference will increase linearly with the length of the contacted area. This is shown in Figure 9B.

A third simple way in which the curvatures can be compared is by means of height differences. Then one would expect to find a constant threshold in terms of 
A Model 1: constant curvature

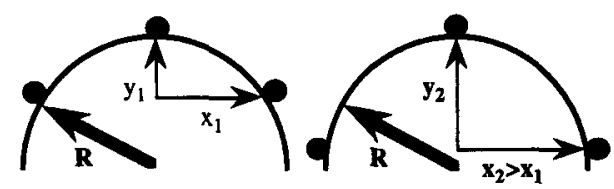

predicts: $\mathbf{y}_{2}>y_{1}$

B Model 2: constant attitude difference

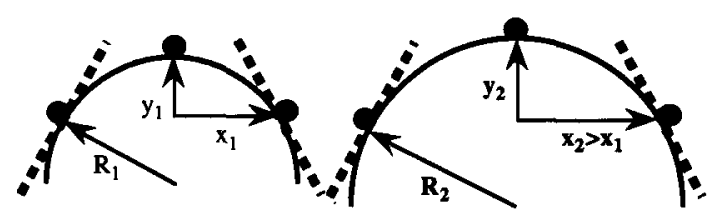

predicts: $R_{2}>R_{1}$ and $y_{2}>y_{1}$

C Model 3: constant height difference
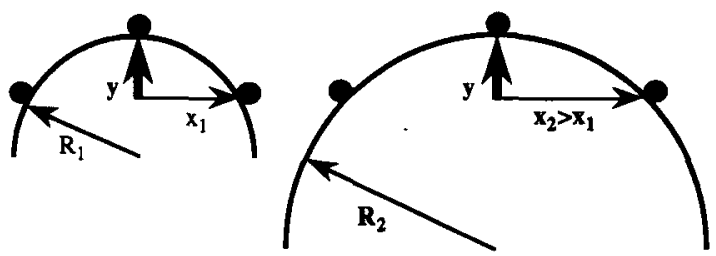

predicts: $\mathbf{R}_{\mathbf{2}}>\mathbf{R}_{\mathbf{1}}$

Figure 8. Schematic representations of the three different models. Two situations are compared: In situation 1 (left), the length over which the strip is touched is shorter than in situation 2 (right). In situation 1 (2), the strip, which is a circular curved stimulus, is touched over a length, $2 x_{1}\left(2 x_{2}\right)$. The discrimination threshold in terms of radius of curvature is represented by $R_{1}\left(R_{2}\right)$ and in terms of base-to-peak height difference by $y_{1}\left(y_{2}\right)$. (A) Representation of the model following the principle of a constant threshold in terms of curvature. (B) Model following the principle of a constant threshold in terms of attitude difference. (C) Model following the principle of a constant threshold in terms of height difference.

height differences. This model is depicted in Figure 8C. When the strips are touched over a length $2 x_{1}$ and a height difference of $y$ can just be discriminated, the threshold expressed in units of radius of curvature will be $R_{1}$ (left panel). When the strips are touched over a length $2 x_{2}$ (right panel) that is larger than $2 x_{1}$, the threshold in terms of height differences, $y$, is by definition the same as in the former situation. Thus, the threshold in terms of radius of curvature $R_{2}$ has to be higher than $R_{1}$. In Figure $9 \mathrm{C}$, the predictions in the case of this model are shown. The starting point is a constant threshold in terms of height difference, which leads to the prediction that the thresholds in terms of radius of curvature will increase as a function of length of contact ( $R_{2}$ is larger than $\left.R_{1}\right)$.

Measurements of the length of the contacted area. A photocopy was made of each subject's hand, on which the hand was projected 1 to 1 . The lengths of the contacted areas could be measured from these photocopies because the contact areas of the hand with the (flat) surface of the photocopier show up white in these images. First we estimated the centers of all these contact areas. Because the areas are approximately ellipses, this could be done by determining the midpoints of the long and short axes of the areas. Second, the length between the centers of the outermost areas that touched the strips in a certain placement was measured with a ruler. This value is defined as the center-to-center contact length (see also Figure 7B).

A second measure of the length of the contacted area is the total contact length (see also Figure 7B). These

\section{A Model 1: constant curvature}

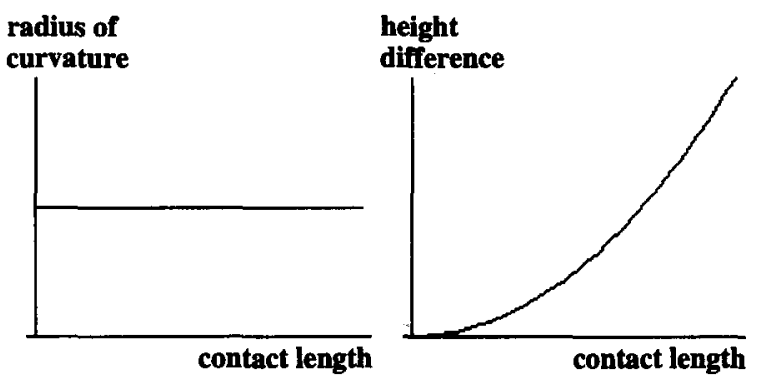

B Model 2: constant attitude difference radius of curvature

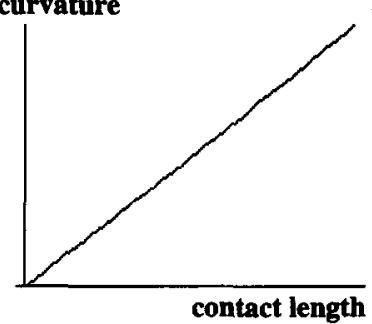

height difference

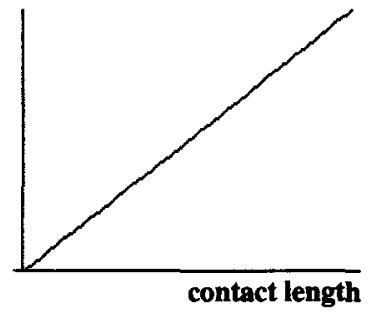

C Model 3: constant height difference radius of curvature

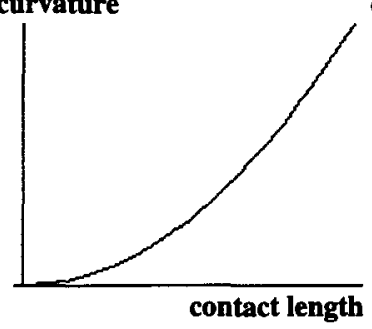

height difference

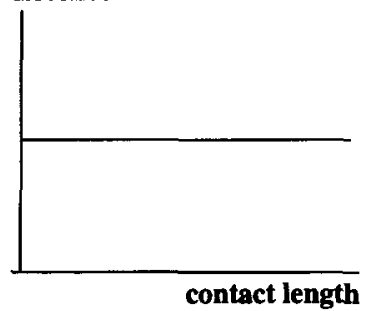

Figure 9. The predictions for the thresholds in terms of radius of curvature ( $R$, figures on the left side) and base-to-peak height difference $(y$, figures on the right side) as a function of the centerto-center contact length ( $2 x$ ). Panels $A, B$, and $C$ in Figure 9 correspond to the three models in panels $A, B$, and $C$ in Figure 8 , respectively. 

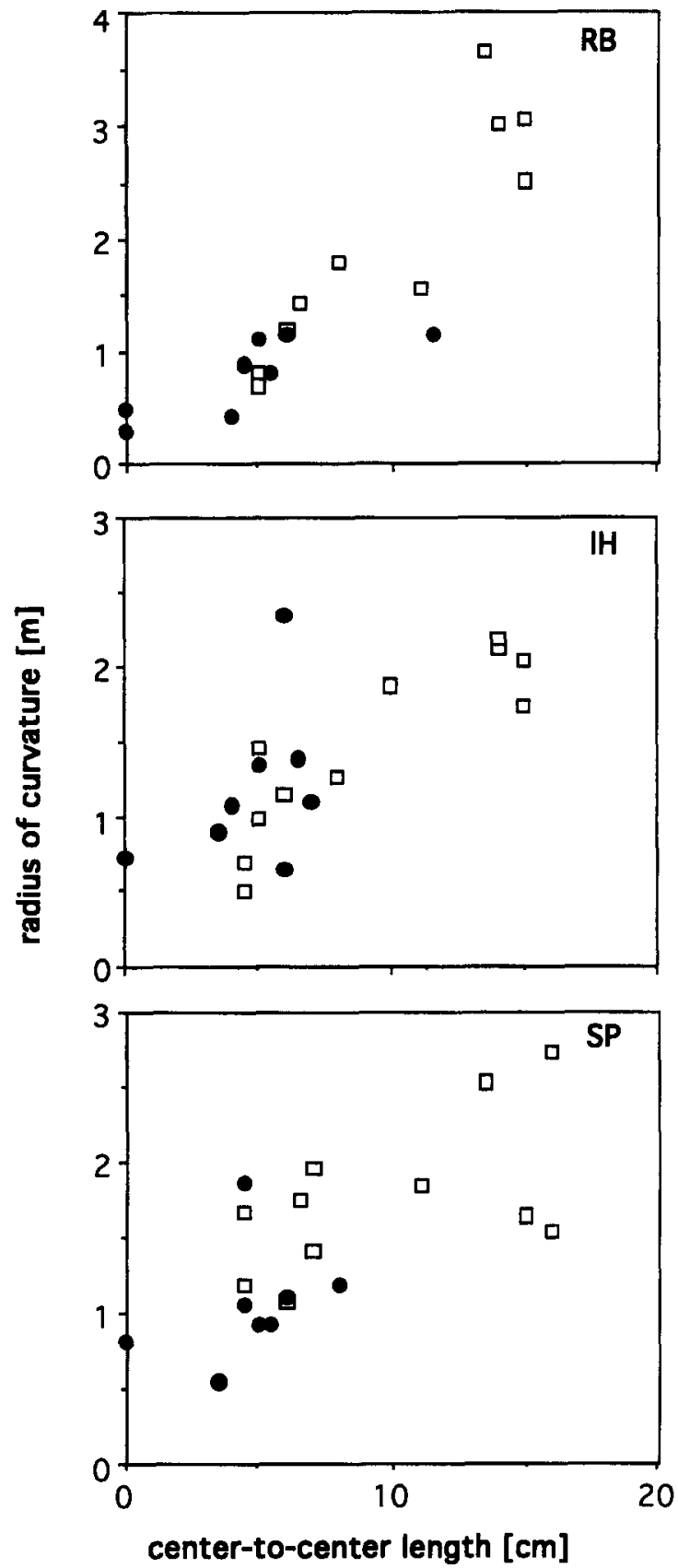

Figure 10. Discrimination thresholds in terms of radius of curvature for all different placements as a function of the center-tocenter length of contact for Experiments 1 (open squares) and 2 (filled circles).

lengths were determined from the photocopies by putting a ruler on a line corresponding to a certain placement of the strips. Then the total contact length, defined as the length between the outermost spots that contact the strips in a certain placement, could be read from the ruler directly.

The measurements were checked by estimations of the contact lengths that had been made while the subjects touched the strips in the experimental setup. The errors due to imprecise positioning were reckoned with in these estimations. The differences between the measurements and estimations were at most $0.5 \mathrm{~cm}$, which is smaller than the differences between the lengths for the different placements. The error due to the shape of the strips, circularly curved and not flat, was calculated as the difference between the length of the arc and the base of the arc that was touched. This error was at most $1 \%$ for the most curved strips and the largest contact lengths, and thus can be neglected. Therefore, the ranking of the values for both the center-to-center contact lengths and the total contact lengths was not influenced by these errors.

\section{Results}

In Figure 10, the discrimination thresholds for Experiment 2 (open squares) and for the flat reference curvature for all palmar conditions of Experiment 1 (filled circles) are shown as a function of center-to-center contact length. The results for Subjects R.B., I.H., and S.P. are shown separately. The thresholds expressed in units of radius of curvature $(\mathrm{m})$, the inverse of the thresholds in units of curvature, are represented on the vertical axis. The thresholds in terms of radius of curvature show an overall increase when length of contact increases. For all 3 subjects, the significance of this relation was tested by computation of the Spearman rank order correlation coefficients, as well as the corresponding significance levels. The values for these coefficients are shown in Table 2 in the second column; the corresponding significance levels (one-tailed $p$ values; $d f=17$ ) are shown in the third column. As can be seen, the increase in the discrimination thresholds expressed in units of radius of curvature with center-to-center length of contact was significant.

We also computed the values of the discrimination thresholds in terms of the base-to-peak height difference over the area over which the stimuli were contacted. These values are represented in Figure 11 for Experiments 1 (open squares) and 2 (filled circles) as a function of center-to-center contact lengths. The results for Subjects R.B., I.H., and S.P. are shown in Figure 11 (the upper, middle, and lower panels, respectively). The discrimination thresholds in terms of height differences show an overall increase with length of contact. The Spearman rank order correlation coefficients and corresponding significance levels (one-tailed $p$ values; $d f=17$ ) for all 3 subjects (Table 2, the fourth and fifth columns, respectively) show that this relation was also significant. Thus when the center-to-center contact length increases, the thresholds both in terms of radius of curvature and in terms of height difference show an overall increase.

The correlation with other variables, instead of centerto-center contact length, was also tested. The thresholds as a function of the total length of contact correlated significantly, too. The correlation coefficients and significance levels (one-tailed $p$ values; $d f=17$ ) for these relations are presented in Table 3 . These correlation coefficients and their significance levels are lower than those in Table 2. Thus, the thresholds correlated less strongly with the total contact length than with the centerto-center contact length. The relations as a function of 

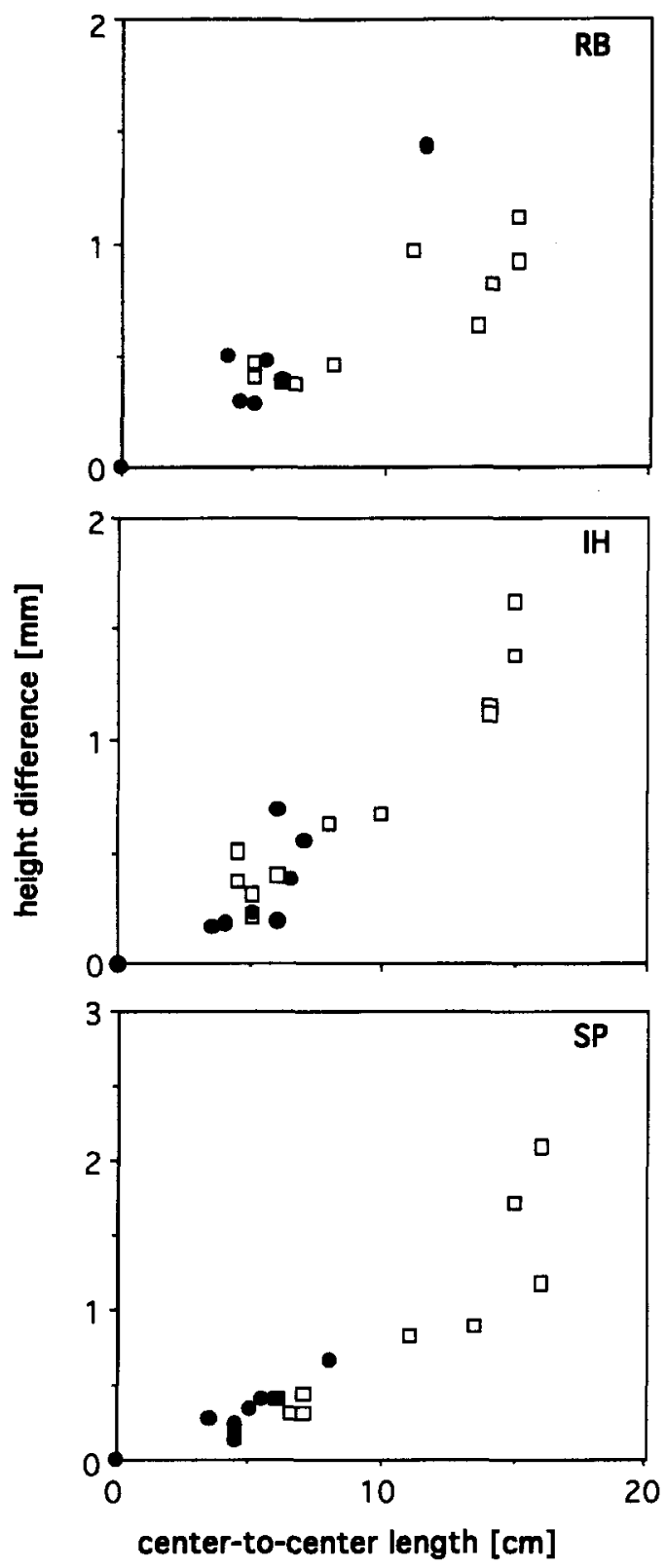

Figure 11. Discrimination thresholds in terms of base-to-peak height difference over the touched part of the stimulus for all different placements as a function of the center-to-center length of contact for Experiments 1 (open squares) and 2 (filled circles).

number of contact areas involved also showed some correlation, but still less than in the case of total contact length. The thresholds as a function of the number of joints involved did not correlate significantly.

\section{Discussion}

The discrimination thresholds in terms of radius of curvature and base-to-peak height difference showed a significant increase (Tables 2 and 3 ) as a function of the center-to-center contact length. These relations are not expected to be significant if the structure of the part of the hand with which the strips are touched is what primarily determines the sensitivity for curvature discrimination. The significance of these relations means that the length over which the strips are contacted, and thus the geometrically available structure, is the major factor determining the sensitivity for curvature discrimination. The fact that both relations increased as a function of contact length means that the best model is the second one, in which the effective stimulus for the discrimination of curved strips is the total change of local surface attitude (compare the left panel of Figure 9B with Figure 10, and the right panel of Figure 9B with Figure 11).

Goodwin et al. (1991) showed that curvature can be discriminated using cutaneous stimulation only. In that case, the local pressure profiles on the skin had to be combined into a representation of curvature. The thresholds they found for the discrimination of a flat surface from a curved surface were $+4.9 / \mathrm{m}$ and $-5.4 / \mathrm{m}$. Their results suggest that this mechanism - comparison of local curvature (our first model) - cannot apply to the discrimination of curved strips with much lower curvatures, namely between $-1.8 / \mathrm{m}$ and $+1.8 / \mathrm{m}$. Our results confirm this. LaMotte and Srinivasan (1993) showed that the activity of slowly adapting cutaneous mechanoreceptors represented the curvatures of the shapes that had been indented into the fingerpads of primates. The curvatures they used were in the range of 79 to $1,260 / \mathrm{m}$ much higher than those we used.

The third mechanism, comparison of curvatures by means of height differences (the zeroth-order geometrical structure), must be triggered by knowledge of relative positions of different parts of the hand. It was found that this mechanism does not apply to our results, and this outcome has been supported by Clark, Burgess, and Chapin (1983), who concluded that humans lack a sense of static position of the fingers.

Our results suggest that local attitudes at locations separated by a certain length are used for static curvature discrimination. The local attitudes represent the firstorder geometrical structure and might be determined from pressure gradients on the skin, relative to some external or internal standard attitude. Furthermore, a combination of the positions of the parts of the hand and the contact areas is needed for the determination of the locations of these attitudes. Finally, the local attitudes and their locations have to be combined somehow into a rep-

Table 2

Values of the Spearman Rank-Order Correlation Coefficients and Significance Levels (one-tailed $p$ values, $d f=17$ ) for the 3 Subjects

\begin{tabular}{lccccc}
\hline & \multicolumn{2}{c}{$R(x)$} & \multicolumn{3}{c}{$y(x)$} \\
\cline { 2 - 3 } \cline { 5 - 6 } Subject & $r_{\mathrm{s}}$ & $p$ & & $r_{s}$ & $p$ \\
\hline R.B. & .919 & $1.310^{-8}$ & .753 & $1.010^{-4}$ \\
I.H. & .720 & $2.510^{-4}$ & .882 & $2.910^{-7}$ \\
S.P. & .632 & $1.910^{-3}$ & .928 & $5.210^{-9}$ \\
\hline
\end{tabular}

Note-These values were calculated for the thresholds in terms of both radius of curvature and base-to-peak height differences as a function of the center-to-center length of contact. 
Table 3

Values of the Spearman Rank-Order Correlation Coefficients and Significance Levels (one-tailed $p$ values, $d f=17$ ) for the 3 Subjects

\begin{tabular}{lccrcc}
\hline & \multicolumn{2}{c}{$R(x)$} & & \multicolumn{2}{c}{$y(x)$} \\
\cline { 2 - 3 } \cline { 5 - 6 } Subject & $r_{s}$ & $p$ & & $r_{s}$ & $p$ \\
\hline R.B. & .822 & $7.910^{-6}$ & & .463 & $2.310^{-2}$ \\
I.H. & .488 & $1.710^{-2}$ & & .856 & $1.510^{-6}$ \\
S.P. & .625 & $2.010^{-3}$ & & .821 & $8.210^{-6}$ \\
\hline
\end{tabular}

Note-These values were calculated for the thresholds in terms of both radius of curvature and base-to-peak height differences as a function of the total length of contact.

resentation of the curvature of the stimulus. The manner in which this happens needs to be investigated further.

\section{GENERAL DISCUSSION}

The results of Experiment 1 show that cutaneous stimulation is important in the static discrimination of the curvature of strips. This follows from the facts that performance was poorer in the dorsal than in the palmar conditions and that the resolution of the cutaneous stimulation was reduced dorsally, whereas the resolution of the kinesthetic stimulation was constant.

Cutaneous stimulation may contribute to the discrimination of curved strips in at least three possible ways. First, cutaneous stimulation probably serves an important supportive or facilitatory role in the sensation of joint positions and movements. This role of cutaneous stimu- lation has been suggested by Clark et al. (1983) and Gandevia, McCloskey, and Burke (1992). Second, cutaneous stimulation may be of significance for evaluating the positions of joints via detection of the amount of skin stretch as well as shearing forces (Vallbo \& Johansson, 1978). Third, cutaneous stimulation can yield knowledge about the curvature of the strips through pressure gradients by which the local attitudes on the different areas of contact can be known. Several researchers have shown that the slowly adapting cutaneous mechanoreceptors system is sensitive to discontinuities and curvature in spatial form (Johnson et al., 1996; LaMotte \& Srinivasan, 1993; Vallbo, 1996). It has not been tested explicitly whether this system is also sensitive to pure attitude differences. This mechanism needs to be investigated further.

Overall, we found that the curvature of the reference strip had no significant effect on the discrimination thresholds. Kappers and Koenderink (1996) found that performance was better for larger absolute curvatures. However, the maximum absolute curvature in their experiment, $5.7 / \mathrm{m}$, was much larger than that in our experiment, $1.8 / \mathrm{m}$. Goodwin and Wheat (1992) also found better performance for larger curvatures. They used reference curvatures of $154 / \mathrm{m}$ and $286 / \mathrm{m}$, which are much larger than ours $(-0.8 / \mathrm{m}, 0 / \mathrm{m}$, and $0.8 / \mathrm{m}$ ). In addition, the haptic strategies and the sizes of the curved surfaces showed large differences. In the experiment of Kappers and Koenderink (1996), hand-sized surfaces were touched dynamically. In the investigation of Goodwin and Wheat (1992), surfaces of $19.6 \mathrm{~mm}^{2}$ were touched statically with a fingerpad. In our experiment, the

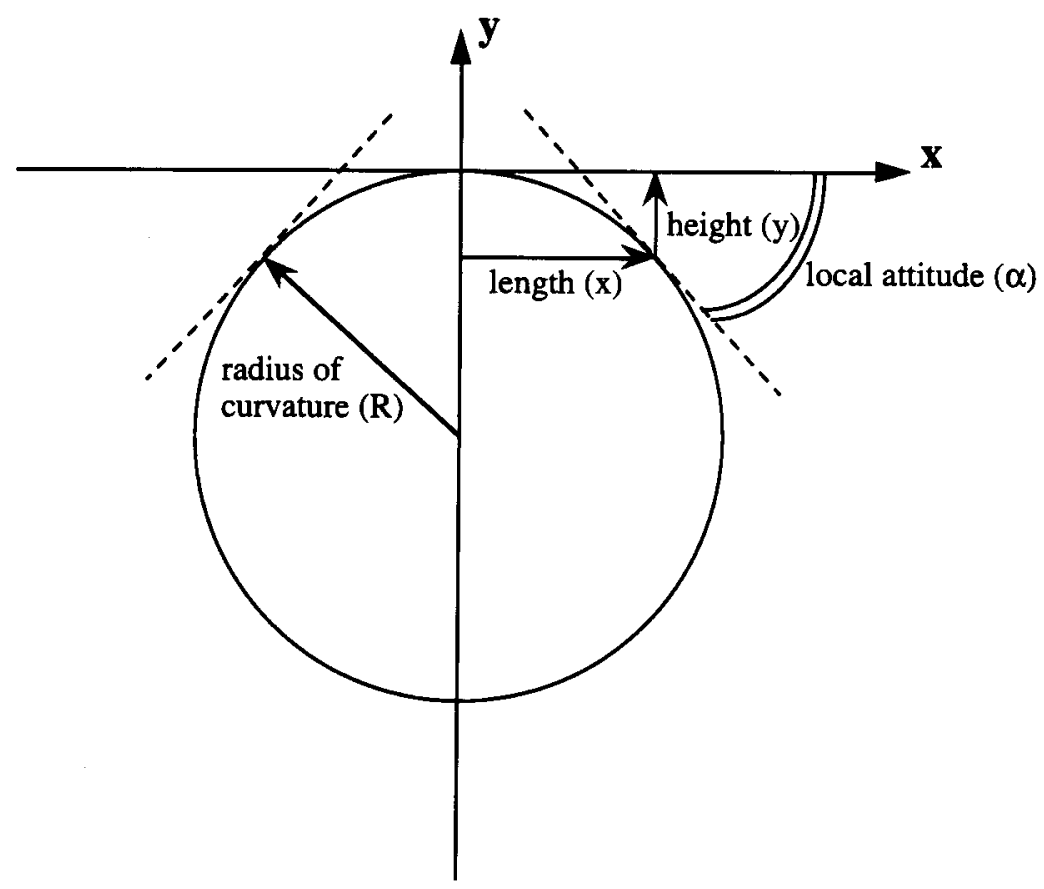

Figure 12. Schematic representation of a part of the hand contacting a strip. The base length of the contacted area is $2 x$. The base-to-peak height difference over that part of the strip is $y$, which is determined by the radius of curvature, $R$, and the length of contact, $2 x$. The local attitude on position $(x, y)$ is $\alpha$. 
$20-\mathrm{cm}$-long strips were touched statically. These dissimilarities, especially those concerning the curvature ranges, probably explain the different results.

Our analysis of the results of Experiments 1 and 2 in terms of the geometrical properties of the stimuli shows that the differences in the thresholds for the different positions are due primarily to the length over which the stimulus was touched. The thresholds, both in terms of radius of curvature and in terms of height difference, show a significant increase as a function of the center-tocenter contact length. Therefore, the best model is one in which the effective stimulus for the discrimination of curved strips is the total change of local surface attitude. Although it remains to be seen how closely the discrimination of curved strips follows this principle, it is surprising to find a correlation like this, especially if we consider the differences between the parts of the hand with which the strips were touched in certain conditions. For instance, the results we found for the discrimination of strips with the palm or with the index finger or with the thumb all conform to a single trend. This model can also explain the result of Experiment 1, namely that the discrimination of curved strips was almost a factor of 2 better when the strips were touched along the fingers than when they were touched perpendicular to the fingers, since the contact length was about twice as large in the first case. This effect of a larger contact area could also explain the difference between the values we found and the much larger values Goodwin et al. (1991) found for the discrimination of curvature with a single fingerpad.

The model suggests that curved strips are discriminated by comparing attitude differences. In research by Gordon and Morison (1982), the effective stimulus for curvature was also found to be the total attitude change over a curved surface. In their experiment, the touching process was very different from the way in which the stimuli were touched in our experiment. Nevertheless, the physical properties of the stimuli on which the discrimination was based seem to be the same, namely attitude differences over the surfaces. They found a value of .009 for the gradient (base-to-peak height divided by the length of the stimulus). It is difficult to fit an exact numerical value to our data, but a global comparison shows that the values we found are of the same order of magnitude. This value corresponds with a total attitude difference over the surface of the strips of $2^{\circ}$. In other words, the alpha in Figure 12 is only about $1^{\circ}$. Thus, when the total attitude difference over the touched part of a curved surface is $2^{\circ}$, it can be discriminated from a flat surface. Moreover, we found that this did not depend on the exact locus of the curve on the palmar side of the hand.

\section{REFERENCES}

Clark, F. J., Burgess, R. C., \& Chapin, J. W. (1983). Humans lack a sense of static-position of the fingers. Society for Neuroscience Abstracts, 9, 1033 .

COREN, S. (1993). The left-hander syndrome. New York: Vintage Books.
CREWDSON, J., \& ZANGwill, O. L. (1940). A note on tactual perception in a blind subject. British Journal of Psychology, 30, 224-229.

Davidson, P. W. (1972). Haptic judgments of curvature by blind and sighted humans. Journal of Experimental Psychology, 93, 43-55.

Davidson, P. W., \& Whitson, T. T. (1974). Haptic equivalence matching of curvature by blind and sighted humans. Journal of Experimental Psychology, 102, 687-690.

Gandevia, S. C., McCloskey, D. I., \& Burke, D. (1992). Kinaesthetic signals and muscle contraction. Trends in Neurosciences, 15, 62-65.

Goodwin, A. W., John, K. T., \& Marceglia, A. H. (1991). Tactile discrimination of curvature by humans using only cutaneous information from the fingerpads. Experimental Brain Research, 86, 663672.

GOODWIN, A. W., \& WHEAT, H. E. (1992). Human tactile discrimination of curvature when contact area with the skin remains constant. Experimental Brain Research, 88, 447-450.

GORDON, I. E., \& MoRISON, V. (1982). The haptic perception of curvature. Perception \& Psychophysics, 31, 446-450.

HuNTER, I. M. L. (1954). Tactile-kinaesthetic perception of straightness in blind and sighted humans. Quarterly Journal of Experimental Psychology, 6, 149-154.

Johnson, K. O., Hsiao, S. S., \& Twombly, I. A. (1996). Neural mechanisms of tactile form recognition. In M. S. Gazzaniga (Ed.), The cognitive neurosciences (pp. 253-267). Cambridge, MA: MIT Press.

Kappers, A. M. L., \& Koenderink, J. J. (1996). Haptic unilateral and bilateral discrimination of curved surfaces. Perception, 25, 739749

Kappers, A. M. L., KoenderinK, J. J., \& te PAs, S. F. (1994). Haptic discrimination of doubly curved surfaces. Perception, 23, 1483-1490.

LaMotte, R. H., \& SRinivasan, M. A. (1993). Responses of cutaneous mechanoreceptors to the shape of objects applied to the primate fingerpad. Acta Psychologica, 84, 41-51.

LEDERMAN, S. J., \& KLATZKY, R. L. (1987). Hand movements: A window into haptic object recognition. Cognitive Psychology, 19, 342-368.

Loomis, J. M., \& Lederman, S. J. (1986). Tactual perception. In K. R. Boff, L. Kaufman, \& J. P. Thomas (Eds.), Handbook of perception and human performance: Vol. 2. Cognitive processes and performance (pp. 31.1-31.41). New York: Wiley.

Macmillan, N. A., \& Creelman, C. D. (1991). Detection theory: A user's guide. Cambridge: Cambridge University Press.

Press, W. H., Flannery, B. P., Teukolsky, S. A., \& Vetterling, W. T. (1988). Numerical recipes in $C$ (pp. 540-575). Cambridge: Cambridge University Press.

Rubin, E. (1936). Haptische Untersuchungen [Haptic research]. Acta Psychologica, 1, 285-380.

VALLBO, A. B. (1996). Single-afferent neurons and somatic sensation in humans. In M. S. Gazzaniga (Ed.), The cognitive neurosciences (pp. 237-252). Cambridge, MA: MIT Press.

VALLBO, A. B., \& Johansson, R. S. (1978). The tactile sensory innervation of the glabrous skin of the human hand. In G. Gordon (Ed.), Active touch (pp. 29-54). Oxford: Pergamon.

Vogels, I. M. L. C., Kappers, A. M. L., \& Koenderink, J. J. (1996) Haptic after-effect of curved surfaces. Perception, 25, 109-119.

WEBER, E. H. (1978). De subtilitate tactus [The sense of touch]. In H. E. Ross, D. J. Murray, \& J. D. Mollon (Eds.), The sense of touch (pp. 19-138). London: Academic Press. (Original work published 1834)

WEINSTEIN, S. (1968). Intensive and extensive aspects of tactile sensitivity as a function of body part, sex, and laterality. In D. R. Kenshalo (Ed.), The skin senses (pp. 195-218). Columbus, OH: C. C. Thomas.

\section{APPENDIX}

The mathematical derivation for the base-to-peak height difference $(y)$ over a certain contacted area of a circularly curved strip follows from the definition of the circle with a radius of $R=1 / k$, with $k$ the curvature (Figure 10 ): 


$$
x^{2}+(R-y)^{2}=R^{2}
$$

from which it follows that

and

$$
R=\frac{x^{2}+y^{2}}{2 y}
$$

$$
y=R-\left(R^{2}-x^{2}\right)^{1 / 2},
$$

in which $x$ is half of the base length of the contacted area and $y$ is the base-to-peak height difference over this region. Thus the Taylor series starts with a quadratic term:

$$
y=x^{2} / 2 R+\ldots
$$

The local attitude $(\alpha)$ at a certain position $(x, y)$ on a strip with a radius of curvature $R$ is determined by the first derivative of this function:

through

$$
\frac{d y}{d x}=\frac{x}{\left(R^{2}-x^{2}\right)^{1 / 2}}=x / R+\ldots
$$

$$
\alpha=\arctan (d y / d x)=x / R+\ldots
$$

Thus these Taylor series start with a linear term.

The derivation of the functions that describe the models (Figures 8-9) follows from the combination of these general expressions and the assumptions on which the models are based. Given the geometry of the stimuli (which is described by the expressions above) and some assumption about the mechanism that determines the discrimination thresholds (for which we choose three limit cases that determine the three models), pre- dictions are made for the thresholds as a function of contact length.

\section{Model 1}

Assumption: The threshold in terms of the radius of curvature is constant. The Taylor series for the base-to-peak height difference $y$ starts with a quadratic term $x^{2} / 2 R$, so if the radius of curvature is a constant at threshold level, it follows that the threshold in terms of base-to-peak height difference has to increase quadratically as a function of contact length.

\section{Model 2}

Assumption: The threshold in terms of local attitudes is constant. The ratio base-to-peak height difference/contact length has to be constant in that case. The Taylor series for the local attitude starts with a linear term $x / R$. Thus it follows that the thresholds in terms of radius of curvature and in terms of baseto-peak height difference have to increase linearly as a function of contact length.

\section{Model 3}

Assumption: The threshold in terms of base-to-peak height difference is constant. From the equation for the radius of curvature, it follows that the threshold in terms of the radius of curvature has to increase quadratically as a function of the contact length in this case.

(Manuscript received June 10, 1996;

revision accepted for publication November 20, 1996.) 